\title{
Tunable quantum interference effect on magnetoconductivity in few-layer black phosphorus
}

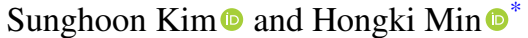 \\ Department of Physics and Astronomy, Seoul National University, Seoul 08826, Korea
}

(Received 5 December 2019; revised manuscript received 29 April 2020; accepted 7 May 2020; published 28 May 2020)

\begin{abstract}
In this Rapid Communication, we develop a systematic weak localization and antilocalization theory fully considering the anisotropy and Berry phase of the system, and apply it to various phases of few-layer black phosphorus (BP), which has a highly anisotropic electronic structure with an electronic gap size tunable even to a negative value. The derivation of a Cooperon ansatz for the Bethe-Salpeter equation in a general anisotropic system is presented, revealing the existence of various quantum interference effects in different phases of few-layer BP, including a crossover from weak localization to antilocalization. We also predict that the magnetoconductivity at the semi-Dirac transition point will exhibit a nontrivial power-law dependence on the magnetic field, while following the conventional logarithmic field dependence of two-dimensional systems in the insulator and Dirac semimetal phases. Notably, the ratio between the magnetoconductivity and Boltzmann conductivity turns out to be independent of the direction, even in strongly anisotropic systems. Finally, we discuss the tunability of the quantum corrections of few-layer BP in terms of the symmetry class of the system.
\end{abstract}

DOI: 10.1103/PhysRevResearch.2.022045

Introduction. The presence of disorder enriches the physics of mesoscopic systems [1-4]. One of the remarkable effects of disorder on transport properties is a negative correction to the dc conductivity, so-called weak localization (WL). WL arises as electrons obtain an enhanced probability of backscattering due to a quantum interference effect. The theoretical description of the WL effect is accessible in a Feynman diagram formalism [5-8], and its signal has been explored extensively through experiments $[9,10]$. In contrast, electrons carrying a $\pi$ Berry phase result in a positive quantum correction to the dc conductivity, which is referred to as weak antilocalization (WAL). WAL is attributed to a suppressed probability of backscattering due to an additional minus sign by the $\pi$ Berry phase. The WAL effect has been studied intensely in topological states of matter with the $\pi$ Berry phase, such as graphene [11-16], topological semimetals [17-21], and surface states of topological insulators [22-24]. Such materials show complex quantum interference corrections due to the competition between the WL and WAL effects [11,12,21].

Few-layer black phosphorus (BP), a two-dimensional (2D) van der Waals material which has been studied intensely both theoretically [25-37] and experimentally [38-46], is expected to show nontrivial quantum interference effects due to strong anisotropy and a tunable electronic structure. Few-layer BP has a direct band gap [Fig. 1(a)], which can be tuned by external perturbations, such as strain [25], pressure [42], electric gating [27,33], and chemical doping [29,31]. Such modula-

\footnotetext{
*hmin@snu.ac.kr

Published by the American Physical Society under the terms of the Creative Commons Attribution 4.0 International license. Further distribution of this work must maintain attribution to the author(s) and the published article's title, journal citation, and DOI.
}

tions can close the band gap, resulting in a semi-Dirac transition point (SDTP) with a combination of linear and quadratic dispersions [Fig. 1(b)]. These modulations can even induce a band inversion, leading to the Dirac semimetal (DSM) phase with linear dispersions around the nodes [Fig. 1(c)]. Although there have been a few experimental studies on the quantum interference effects in few-layer BP [43-46], a theoretical approach on each phase has been elusive due to its nontrivial anisotropy, which cannot be described by a simple model with different effective masses in each direction. Thus, a further systematic formalism of the quantum interference theory is called for.

In this Rapid Communication, we develop a general framework for the quantum interference effect, which captures the anisotropy and Berry phase effect of the system. This is achieved by deriving a Cooperon ansatz which applies to a general system with an anisotropic energy dispersion. By applying this framework to the various phases of few-layer BP, we find that the insulator phase and SDTP have the anisotropic WL corrections, while the DSM phase can host either WL or WAL arising from internode and intranode scatterings, respectively. We also estimate the effect of a magnetic field on the quantum corrections in each phase, predicting a nontrivial power-law dependence on the magnetic field at the SDTP. Notably, we show that the ratio between the magnetoconductivity and Boltzmann conductivity is independent of the direction, even in highly anisotropic systems. Finally, we discuss the tunability of the quantum interference effect in few-layer BP, with comments on the symmetry class.

Model. The low-energy effective Hamiltonian of few-layer $\mathrm{BP}$ is given by $[31,34,36,37]$

$$
H=\left(\frac{\hbar^{2} k_{x}^{2}}{2 m^{*}}+\frac{E_{\mathrm{g}}}{2}\right) \sigma_{x}+\hbar v_{y} k_{y} \sigma_{y}
$$


(a)

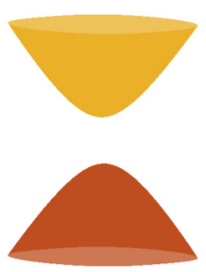

(b)
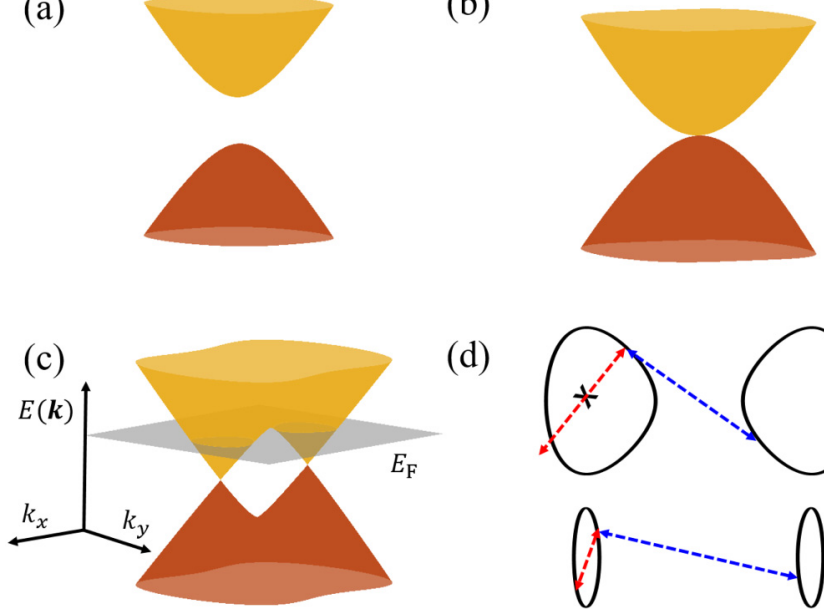

(d)

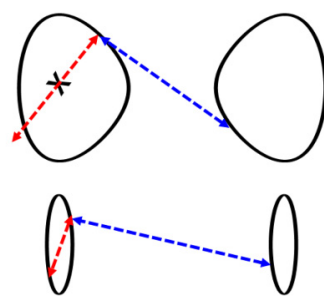

FIG. 1. Electronic structure of few-layer BP in the (a) insulator phase, (b) semi-Dirac transition point (SDTP), and (c) Dirac semimetal (DSM) phase. (d) The Fermi surfaces of the DSM phase. At a sufficiently low Fermi energy (lower panel), a momentum state has time-reversed counterparts both within the node and in the opposite node. Thus, the quantum interference effect is contributed by two types of scatterings: intranode (red dashed arrow) and internode (blue dashed arrow) scatterings. As the Fermi energy increases (upper panel), the Fermi surface is distorted and the time-reversal symmetry around a node is broken. Thus, the quantum interference effect via intranode scattering is suppressed.

where $m^{*}$ is the effective mass along the zigzag $(x)$ direction, $E_{\mathrm{g}}$ is the band gap, $v_{y}$ is the velocity along the armchair $(y)$ direction, and $\sigma_{x, y}$ are the Pauli matrices. The corresponding en-

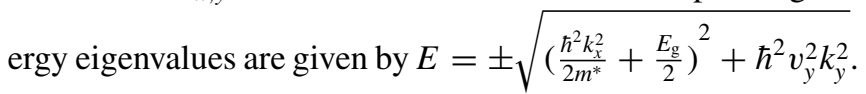
Without band gap tuning, the Hamiltonian has a direct band gap $\left(E_{\mathrm{g}}>0\right)$ and few-layer BP is in the gapped insulator phase [Fig. 1(a)]. At $E_{\mathrm{g}}=0$, the Hamiltonian has a band touching point at $\left(k_{x}, k_{y}\right)=(0,0)$ and few-layer $\mathrm{BP}$ is in the SDTP [Fig. 1(b)]. At this point, the energy dispersion is linear in the armchair direction and quadratic in the zigzag direction. As the band gap decreases further to a negative value $\left(E_{\mathrm{g}}<0\right)$, few-layer BP becomes the DSM phase with two nodes at $\boldsymbol{K}^{ \pm}=\left( \pm \sqrt{\frac{m^{*}\left|E_{\mathrm{g}}\right|}{\hbar^{2}}}, 0\right)$ [Fig. 1(c)]. At a sufficiently low Fermi energy satisfying $E_{\mathrm{F}} \ll\left|E_{\mathrm{g}}\right|$, the Hamiltonian near each node can be linearized as

$$
\begin{aligned}
H & = \pm \hbar v_{x}\left(k_{x}-K_{x}^{ \pm}\right) \sigma_{x}+\hbar v_{y} k_{y} \sigma_{y} \\
& \equiv \hbar v_{0} \kappa\left( \pm \cos \phi_{\kappa} \sigma_{x}+\sin \phi_{\kappa} \sigma_{y}\right),
\end{aligned}
$$

where $v_{x}=\sqrt{\frac{\left|E_{g}\right|}{m^{*}}}$ is the velocity along the zigzag direction. For later convenience, we adopt the parametrization $v_{0} \kappa \cos \phi_{\kappa}=v_{x}\left(k_{x}-K_{x}^{ \pm}\right)$and $v_{0} \kappa \sin \phi_{\kappa}=v_{y} k_{y}$, where $\kappa$ is an effective momentum measured from the nodes. In this work, we neglect the effects of spin-orbit coupling due to its negligible size in few-layer BP.

The quantum correction is determined not only by the electronic structure, but also by the type of impurity. The quantum interference effect is only induced by static and nonmagnetic impurities as it is destroyed by nonstatic or (a)

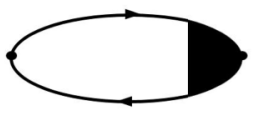

(b)

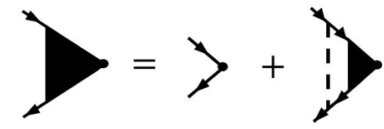

(c)

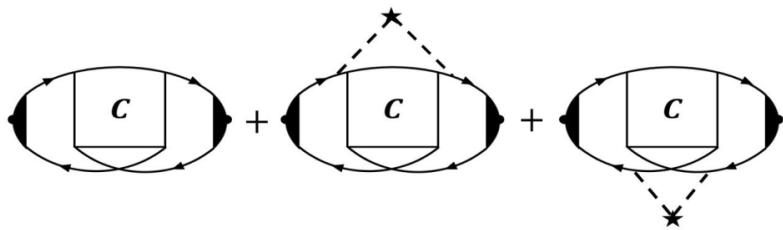

(d)
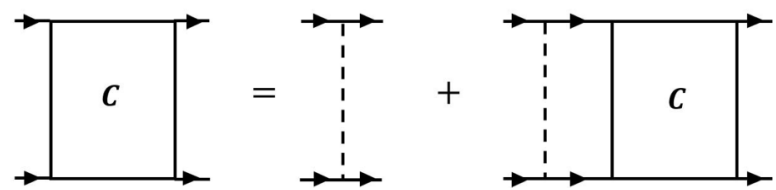

FIG. 2. Feynman diagrams describing the corrections to the dc conductivity. (a) The current-current correlation function supplemented with the ladder vertex correction gives results equivalent to the Boltzmann transport theory. (b) The ladder vertex correction satisfies the self-consistent Dyson's equation. (c) The quantum correction to the dc conductivity is mostly contributed by a bare Hikami box and two dressed Hikami boxes. (d) The Cooperon operator obeys the self-consistent Bethe-Salpeter equation.

magnetic impurities [3,4]. Another aspect we should consider is the range of scattering. At low Fermi energies, regardless of the range of scattering, an electron in the insulator phase and SDTP has a single time-reversed counterpart on the whole Fermi surface. In contrast, for the DSM phase we consider two types of scatterings: intranode and internode scatterings [Fig. 1(d)]. In the $E_{\mathrm{F}} \ll E_{\mathrm{g}}$ limit, intranode scattering may occur by long-range impurities, whereas internode scattering may arise from short-range impurities, such as lattice vacancies. The relative strengths of intranode and internode scatterings lead to competition between the WAL and WL effects in the DSM phase, as will be discussed later.

Diagrammatic approach. In a diagrammatic approach, effects of weak disorder on transport can be studied by incorporating relevant corrections into the current-current correlation function with disorder-averaged Green's functions $[47,48]$. Calculating the self-energy up to the first-order Born approximation, we obtain the quasiparticle lifetime $\left(\tau_{k}^{\mathrm{qp}}\right)^{-1}=$ $\frac{1}{\mathcal{V}} \sum_{\boldsymbol{k}^{\prime}} W_{\boldsymbol{k}^{\prime}, \boldsymbol{k}}$, where $\mathcal{V}$ is the volume of the system and $W_{\boldsymbol{k}^{\prime}, \boldsymbol{k}}$ is the transition rate from $\boldsymbol{k}$ to $\boldsymbol{k}^{\prime}$. Note that for impurity scattering, the transition rate is given by $W_{\boldsymbol{k}^{\prime}, \boldsymbol{k}}=\frac{2 \pi n_{\text {imp }}}{\hbar}\left|V_{\boldsymbol{k}^{\prime}, \boldsymbol{k}}\right|^{2} \delta\left(\xi_{\boldsymbol{k}}-\right.$ $\xi_{\boldsymbol{k}^{\prime}}$, where $n_{\text {imp }}$ is the impurity density, $V_{\boldsymbol{k}^{\prime}, \boldsymbol{k}}=\left\langle\boldsymbol{k}^{\prime}|V| \boldsymbol{k}\right\rangle$ is the matrix element of the scattering potential $V$, and $\xi_{k} \equiv \varepsilon_{k}-\mu$ is the energy of an electron with respect to the chemical potential $\mu$. The ladder vertex correction combined with the first-order Born approximation is the leading impurity correction to the current vertex [Fig. 2(a)], which gives results equivalent to those of the semiclassical Boltzmann transport theory. The ladder vertex correction obeys the self-consistent Dyson's equation [Fig. 2(b)], and for isotropic systems, it gives a well-known result with the $(1-\cos \theta)$ factor in the inverse transport relaxation time [49], suppressing the low-angle scattering contribution to transport. In an anisotropic system, the relation is generalized to a coupled integral equation, 
which relates transport relaxation times at different states. In a $d$-dimensional anisotropic system, it reads $[36,50,51]$

$$
1=\int \frac{d^{d} k^{\prime}}{(2 \pi)^{d}} W_{\boldsymbol{k}^{\prime}, k}\left(\tau_{\boldsymbol{k}}^{(i)}-\frac{v_{\boldsymbol{k}^{\prime}}^{(i)}}{v_{\boldsymbol{k}}^{(i)}} \tau_{\boldsymbol{k}^{\prime}}^{(i)}\right),
$$

where $\tau_{k}^{(i)}$ and $v_{k}^{(i)}=\frac{1}{\hbar} \frac{\partial \varepsilon_{k}}{\partial k_{i}}$ are the transport relaxation time and velocity, respectively, along the $i$ th direction. The ladder vertex correction modifies the velocity vertex along the $i$ th direction as $\tilde{v}_{\boldsymbol{k}}^{(i)}=v_{\boldsymbol{k}}^{(i)} \tau_{\boldsymbol{k}}^{(i)} / \tau_{\boldsymbol{k}}^{\mathrm{qp}}$ [51]. In the zero-temperature limit, the ladder approximation yields the dc conductivity in the semiclassical Boltzmann regime as

$$
\sigma_{i j}^{\mathrm{B}}=g e^{2} \int \frac{d^{d} k}{(2 \pi)^{d}} \delta\left(\xi_{\boldsymbol{k}}\right) v_{\boldsymbol{k}}^{(i)} v_{\boldsymbol{k}}^{(j)} \tau_{\boldsymbol{k}}^{(j)},
$$

where $g$ is the degeneracy of the system and the superscript B denotes the Boltzmann conductivity.

Consideration of further diagrams, called maximally crossed diagrams, leads to the quantum interference correction [Figs. 2(c) and 2(d)]. The quantum correction can be boiled down to three leading terms referred to as a bare Hikami box and two dressed Hikami boxes [12]. The Hikami boxes can be computed by the Cooperon operator, which obeys the following Bethe-Salpeter equation [3,4] (in the following, we omit $\hbar$ for simplicity),

$$
\begin{aligned}
C_{\boldsymbol{Q}}^{\mathrm{AR}}\left(\boldsymbol{k}, \boldsymbol{k}^{\prime}\right)= & n_{\mathrm{imp}} V_{\boldsymbol{k}^{\prime}, \boldsymbol{k}} V_{-\boldsymbol{k}^{\prime},-\boldsymbol{k}}+\frac{n_{\mathrm{imp}}}{\mathcal{V}} \sum_{\boldsymbol{p}} V_{\boldsymbol{p}, \boldsymbol{k}} V_{-\boldsymbol{p},-\boldsymbol{k}} C_{\boldsymbol{Q}}^{\mathrm{AR}}\left(\boldsymbol{p}, \boldsymbol{k}^{\prime}\right) \\
& \times G^{\mathrm{A}}(\boldsymbol{p}, 0) G^{\mathrm{R}}(\boldsymbol{Q}-\boldsymbol{p}, 0),
\end{aligned}
$$

where $C_{\boldsymbol{Q}}^{\mathrm{AR}}\left(\boldsymbol{k}, \boldsymbol{k}^{\prime}\right)$ is the Cooperon with the momentum $\boldsymbol{Q}=$ $\boldsymbol{k}+\boldsymbol{k}^{\prime}$, and the superscripts A and $\mathrm{R}$ represent the advanced and retarded functions, respectively. Note that frequencies in the Cooperon are set to zero as we focus on the dc conductivity. The retarded and advanced Green's functions are $G^{\mathrm{R}, \mathrm{A}}(\boldsymbol{p}, \xi)=\left[\xi-\xi_{\boldsymbol{p}} \pm i / 2 \tau_{\boldsymbol{p}}^{\mathrm{qp}}\right]^{-1}$. As the Green's functions near the Fermi surface contribute mostly to the momentum summation in the right-hand side of Eq. (5), we can perform the $\xi_{\boldsymbol{p}}$ integral separately as $\int d \xi_{p} G^{\mathrm{A}}(\boldsymbol{p}, 0) G^{\mathrm{R}}(\boldsymbol{Q}-\boldsymbol{p}, 0)=$ $2 \pi i\left[\boldsymbol{Q} \cdot \boldsymbol{v}_{\boldsymbol{p}}+\frac{i}{2}\left(\frac{1}{\tau_{p}^{\mathrm{qp}}}+\frac{1}{\tau_{p-\boldsymbol{Q}}^{\mathrm{qp}}}\right)\right]^{-1}$ [48]. Using the Ward identities in anisotropic systems [51], we rewrite the denominator of the right-hand side of the $\xi_{p}$ integral as

$$
\boldsymbol{Q} \cdot \boldsymbol{v}_{\boldsymbol{p}}+\frac{i}{2}\left(\frac{1}{\tau_{\boldsymbol{p}}^{\mathrm{qp}}}+\frac{1}{\tau_{\boldsymbol{p}-\boldsymbol{Q}}^{\mathrm{qp}}}\right)=\frac{1}{\tau_{\boldsymbol{p}}^{\mathrm{qp}}}\left[i+f_{\boldsymbol{Q}}(\boldsymbol{p})\right],
$$

where $f_{\boldsymbol{Q}}(\boldsymbol{p}) \equiv \sum_{i} Q^{(i)} v_{p}^{(i)} \tau_{\boldsymbol{p}}^{(i)}$. Accordingly, we have the Bethe-Salpeter equation in anisotropic systems as follows,

$$
\begin{aligned}
C_{\boldsymbol{Q}}^{\mathrm{AR}}\left(\boldsymbol{k}, \boldsymbol{k}^{\prime}\right) \approx & n_{\mathrm{imp}} V_{\boldsymbol{k}^{\prime}, \boldsymbol{k}} V_{-\boldsymbol{k}^{\prime},-\boldsymbol{k}} \\
& +\frac{2 \pi n_{\mathrm{imp}}}{\mathcal{V}} \sum_{\boldsymbol{p}} \delta\left(\xi_{\boldsymbol{p}}\right) V_{\boldsymbol{p}, \boldsymbol{k}} V_{-\boldsymbol{p},-\boldsymbol{k}} C_{\boldsymbol{Q}}^{\mathrm{AR}}\left(\boldsymbol{p}, \boldsymbol{k}^{\prime}\right) \\
& \times \tau_{\boldsymbol{p}}^{\mathrm{qP}}\left[1+i f_{\boldsymbol{Q}}(\boldsymbol{p})-f_{\boldsymbol{Q}}^{2}(\boldsymbol{p})\right],
\end{aligned}
$$

where the Cooperon diverges as $Q \rightarrow 0$, and thus we ignore terms of order higher than $Q^{2}$. For the detailed derivations, see the Supplemental Material [52]. Importantly, we capture the full anisotropy of the system by introducing $f_{Q}(p)$, which is determined by the anisotropic velocities and transport relaxation times on the Fermi surface.

The Bethe-Salpeter equation in Eq. (7) can be solved selfconsistently by collecting the most divergent contribution in the $Q \rightarrow 0$ limit $[11,17,20]$. Alternatively, we directly derive the following Cooperon ansatz by computing the average of Eq. (7) on the Fermi surface [52],

$$
C_{\boldsymbol{Q}}^{\mathrm{AR}}\left(\boldsymbol{k}, \boldsymbol{k}^{\prime}\right)=\frac{\left(2 \pi N_{0} \tau_{\boldsymbol{k}}^{\mathrm{qp}} \tau_{\boldsymbol{k}^{\prime}}^{\mathrm{qp}}\right)^{-1}}{\sum_{i, j} D_{i j} Q_{i} Q_{j}} F\left(\boldsymbol{k}, \boldsymbol{k}^{\prime}\right),
$$

where $N_{0}$ is the density of states at the Fermi surface and $D_{i j}$ are the diffusion coefficients defined by

$$
D_{i j}=\frac{1}{N_{0} \mathcal{V}} \sum_{\boldsymbol{p}} \delta\left(\xi_{\boldsymbol{p}}\right) v_{\boldsymbol{p}}^{(i)} v_{\boldsymbol{p}}^{(j)} \tau_{\boldsymbol{p}}^{(i)} \tau_{\boldsymbol{p}}^{(j)}\left(\tau_{\boldsymbol{p}}^{\mathrm{qp}}\right)^{-1} .
$$

It is worth noting that the ansatz in Eq. (8) includes the electronic structure-dependent phase factor $F\left(\boldsymbol{k}, \boldsymbol{k}^{\prime}\right)$ which is defined via $V_{-\boldsymbol{k}^{\prime},-\boldsymbol{k}} \equiv V_{\boldsymbol{k}^{\prime}, \boldsymbol{k}}^{*} F\left(\boldsymbol{k}, \boldsymbol{k}^{\prime}\right)$ [52]. The ansatz is valid as long as the composition property $F\left(\boldsymbol{k}_{1}, \boldsymbol{k}_{3}\right)=$ $F\left(\boldsymbol{k}_{1}, \boldsymbol{k}_{2}\right) F\left(\boldsymbol{k}_{2}, \boldsymbol{k}_{3}\right)$ holds, as in the case of the various phases of few-layer BP. For the insulator phase and SDTP, $F\left(\boldsymbol{k}, \boldsymbol{k}^{\prime}\right)$ is unity, whereas for the DSM phase, $F\left(\kappa, \kappa^{\prime}\right)=e^{i\left(\phi_{\kappa}-\phi_{\kappa^{\prime}}\right)}$ (unity) for intranode (internode) scattering. Notably, the phase factor reflects the Berry phase of the system which determines the sign of the quantum correction, as will be shown below.

WL and WAL corrections. Using the ansatz in Eq. (8), we compute the total quantum correction $\Delta \sigma_{i i}=\Delta \sigma_{i i}^{\text {bare }}+$ $2 \Delta \sigma_{i i}^{\text {dressed }}$ for each phase [52]. For the insulator phase and SDTP, we find the following WL correction,

$$
\frac{\Delta \sigma_{i i}}{\sigma_{i i}^{\mathrm{B}}}=-\frac{1}{2 \pi^{2} N_{0} D \hbar} \ln \left(\frac{\ell_{\phi}}{\ell_{e}}\right),
$$

where $\ell_{\phi}$ is the phase coherence length, $\ell_{e}$ is the mean free path, and $D \equiv \sqrt{D_{x x} D_{y y}}$. We note that Eq. (10) applies not only to few-layer BP but also to a general 2D anisotropic system regardless of scattering potential [52], with the additional minus sign for the WAL correction. Remarkably, the ratio of the quantum correction to the Boltzmann conductivity is the same irrespective of direction even in strongly anisotropic systems, which also holds in 3D anisotropic systems [52].

For the DSM phase, we compute the quantum corrections due to intranode and internode scatterings, respectively. In this study, for simplicity we consider constant intranode and internode scattering potentials given by $V_{\text {intra }}$ and $V_{\text {inter }}$, respectively. When backscattering is governed by intranode scattering, the Cooperon is given by

$$
C_{Q}^{\mathrm{AR}}\left(\boldsymbol{\kappa}, \boldsymbol{\kappa}^{\prime}\right)=\frac{1}{4\left(\tau_{\mathrm{intra}}^{\mathrm{qp}}\right)^{2}} \frac{n_{\mathrm{imp}} V_{\mathrm{intra}}^{2} e^{i\left(\phi_{\kappa}-\phi_{\boldsymbol{k}^{\prime}}\right)}}{v_{x}^{2} Q_{x}^{2}+v_{y}^{2} Q_{y}^{2}},
$$

where $\left(\tau_{\text {intra }}^{\mathrm{qp}}\right)^{-1}=\frac{n_{\text {imp }} V_{\text {intra }}^{2} E_{\mathrm{F}}}{2 v_{x} v_{y}}$. Importantly, the phase factor $F\left(\boldsymbol{\kappa}, \boldsymbol{\kappa}^{\prime}\right)=e^{i\left(\phi_{\kappa}-\phi_{\kappa^{\prime}}\right)}$ reflects the $\pi$ Berry phase of the DSM phase, which yields the WAL correction to the dc conductivity as

$$
\frac{\Delta \sigma_{i i}}{\sigma_{i i}^{\mathrm{B}}}=\frac{\hbar}{4 \pi E_{\mathrm{F}} \tau_{\text {intra }}^{\mathrm{qp}}} \ln \left(\frac{\ell_{\phi}}{\ell_{\text {intra }}}\right),
$$


where $\ell_{\text {intra }}$ is the mean free path for intranode scattering. On the other hand, the Cooperon for internode scattering is obtained as

$$
C_{Q}^{\mathrm{AR}}\left(\kappa, \kappa^{\prime}\right)=\frac{1}{4\left(\tau_{\text {inter }}^{\mathrm{qp}}\right)^{2}} \frac{n_{\mathrm{imp}} V_{\mathrm{inter}}^{2}}{v_{x}^{2} Q_{x}^{2}+v_{y}^{2} Q_{y}^{2}},
$$

where $\left(\tau_{\mathrm{inter}}^{\mathrm{qp}}\right)^{-1}=\frac{n_{\mathrm{imp}} V_{\text {inter }}^{2} E_{\mathrm{F}}}{2 v_{\mathrm{x}} v_{\mathrm{y}}}$. Thus, internode scattering gives rise to the $\mathrm{WL}$ correction as

$$
\frac{\Delta \sigma_{i i}}{\sigma_{i i}^{\mathrm{B}}}=-\frac{\hbar}{4 \pi E_{\mathrm{F}} \tau_{\text {inter }}^{\mathrm{qp}}} \ln \left(\frac{\ell_{\phi}}{\ell_{\text {inter }}}\right),
$$

where $\ell_{\text {inter }}$ denotes the mean free path for internode scattering.

Magnetoconductivity. Applying an external magnetic field gives an additional phase to each backscattering path, and thus destroys the quantum interference effect [2-4]. In experiments, the phase coherence length can be obtained through the measurement of magnetoconductivity. The magnetoconductivity can be computed using the quantization of Landau levels $[3,20]$. Assuming an external magnetic field along the $z$ direction, we obtain the ratio $P^{(i)}(B) \equiv\left[\Delta \sigma_{i i}(B)-\Delta \sigma_{i i}(0)\right] / \sigma_{i i}^{B}$ for each phase as follows [52],

$$
\begin{aligned}
P_{\mathrm{ins}}^{(i)}(B)= & -\frac{1}{4 \pi^{2} N_{0} D \hbar}\left[\Psi\left(\frac{1}{2}+\frac{\ell_{B}^{2}}{\ell_{e}^{2}}\right)\right. \\
& \left.-\Psi\left(\frac{1}{2}+\frac{\ell_{B}^{2}}{\ell_{\phi}^{2}}\right)-2 \ln \left(\frac{\ell_{\phi}}{\ell_{e}}\right)\right], \\
P_{\mathrm{SDTP}}^{(i)}(B)= & \frac{1}{\pi^{3} D N_{0} \hbar} \int_{\ell_{\phi}^{-1}}^{\ell_{e}^{-1}} d \tilde{Q}_{x} \frac{1}{\tilde{Q}_{x}} \\
& \times \tan ^{-1}\left(\frac{\alpha^{-\frac{2}{3}}}{2^{\frac{2}{3}} \tilde{Q}_{x} \hbar v_{y}} \sqrt{\frac{D_{y y}}{D}}\right), \\
P_{\mathrm{DSM}}^{(i)}(B)= & \frac{\hbar}{8 \pi E_{\mathrm{F}} \tau_{\text {intra }}^{\mathrm{qp}}}\left[\Psi\left(\frac{1}{2}+\frac{\ell_{B}^{2}}{\ell_{\text {intra }}^{2}}\right)\right. \\
& \left.-\Psi\left(\frac{1}{2}+\frac{\ell_{B}^{2}}{\ell_{\phi}^{2}}\right)-2 \ln \left(\frac{\ell_{\phi}}{\ell_{\text {intra }}}\right)\right],
\end{aligned}
$$

where $\Psi(x)$ is the digamma function, and $\alpha \equiv \frac{\sqrt{m^{*}}}{v_{y}} \frac{2 \ell_{B}^{2}}{\hbar^{2} A^{\frac{3}{2}}}$ with $A \approx 1.17325$ [53] and $\ell_{B}=\sqrt{\frac{\hbar c}{4 e B}}$ is the magnetic length. Equation (15c) is obtained for intranode scattering, and the field dependence for internode scattering only differs by the sign, with $\ell_{\text {intra }}\left(\tau_{\text {intra }}^{\mathrm{qp}}\right)$ replaced by $\ell_{\text {inter }}\left(\tau_{\text {inter }}^{\mathrm{qp}}\right)$. For the insulator phase [Fig. 3(a)] and DSM phase [Figs. 3(c) and 3(d)], we find that the field dependence of the magnetoconductivity is well approximated by $P^{(i)}(B) \propto \pm \ln B$, which is in good agreement with the conventional prediction for $2 \mathrm{D}$ systems [5]. However, we predict that the magnetoconductivity of the SDTP will not follow the conventional prediction, but rather will show $P^{(i)}(B) \propto B^{\frac{2}{3}}$ dependence in the weak-field limit $\left(\ell_{\phi} \ll \ell_{B}\right)$, whereas $P^{(i)}(B) \propto B^{\nu}$ dependence in the intermediate-field regime $\left(\ell_{e} \leqslant \ell_{B} \ll \ell_{\phi}\right)$ with the exponent $v$, which depends on the system parameter and can be found numerically [52]. This nontrivial field dependence may be attributed to strong anisotropy in the band dispersion [4], leading to a quantum diffusion which deviates from the $2 \mathrm{D}$
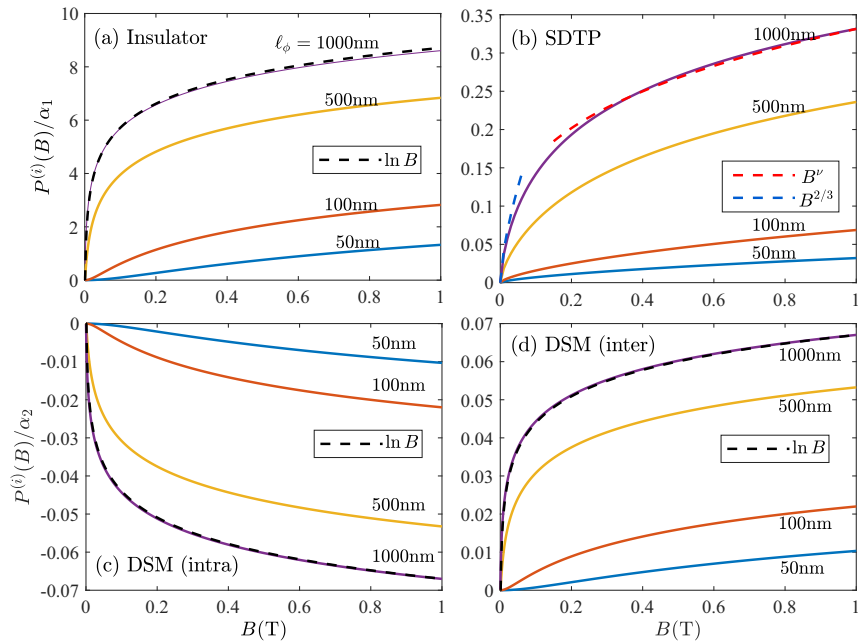

FIG. 3. Ratio of the field-induced change in the magnetoconductivity to the Boltzmann conductivity for various phase coherence lengths with the mean free path $\ell_{e}=10 \mathrm{~nm}$. The ratios for the WL correction in (a) the insulator phase and (b) SDTP are plotted in units of $\alpha_{1}=n_{\text {imp }} V_{\text {imp }}^{2} / \hbar^{2} v_{y}^{2}$, where $V_{\text {imp }}$ denotes the strength of the impurity potential. As for the DSM phase, (c) intranode scattering induces the WAL effect, (d) while internode scattering leads to the WL effect. Both corrections are plotted in units of $\alpha_{2}=n_{\text {imp }} V_{\text {imp }}^{2} / \hbar^{2} v_{x} v_{y}$. As for the field dependence, the black dashed lines denote the $\pm \ln B$ dependence, while the red dashed line and blue dashed line in (b) represent the $B^{v}$ dependence with $v \approx 0.31$ and $B^{2 / 3}$ dependence, respectively. The exponent $v$ is not universal but depends on the system parameter.

behavior. We note that $P^{(i)}(B)$, the ratio of the field-induced change in the magnetoconductivity to the Boltzmann conductivity, is the same irrespective of the direction $i$ for each phase.

Discussion. Our analysis shows that the sign of the quantum correction is determined by the electronic structuredependent phase factor $F\left(\boldsymbol{k}, \boldsymbol{k}^{\prime}\right)$, which reflects the symmetry class of the system $[11,19,54]$. The unity phase factor of the insulator phase and SDTP indicates that the system has spinless time-reversal symmetry, belonging to the orthogonal class. Backscattering induced by a time-reversal operator of this kind leads to WL. As for the DSM phase, in the absence of internode scattering, the phase factor $F\left(\boldsymbol{\kappa}, \boldsymbol{\kappa}^{\prime}\right)=$ $e^{i\left(\phi_{k}-\phi_{k^{\prime}}\right)}$ indicates that the system has time-reversal symmetry around a node without spin-rotational symmetry, belonging to the symplectic class. A system possessing a time-reversal operator of this kind exhibits WAL. In contrast, the presence of internode scattering can induce a crossover from the symplectic to orthogonal class, leading to the corresponding crossover from WAL to WL [11]. Thus, the overall quantum correction in the DSM phase is determined by the dominant scattering mechanism, which depends on the separation between the two nodes [52]. In the $E_{F} \ll\left|E_{\mathrm{g}}\right|$ limit, a large separation between the nodes will suppress the internode scattering rates, and thus the WAL effect might be dominant over the WL effect. In addition, increasing the Fermi energy leads to the distortion of the Fermi surface, suppressing WAL.

Our results can be expanded to multiband systems straightforwardly. By including the interference contribution 
from the time-reversed paths in each band and associated Berry phase effect, we can compute the overall quantum corrections [52].

In this Rapid Communication, we developed a quantum interference theory for anisotropic systems by solving the Bethe-Salpeter equation for the Cooperon operator, fully considering the anisotropy and Berry phase of the system. We elaborated the Cooperon ansatz and diffusion coefficients in a compact and physically intuitive form with transport relaxation times in anisotropic systems, generalizing the previous work by Wölfle et al. [7]. Furthermore, we considered systems with a nontrivial Berry phase, providing a systematic quantum interference theory for both WL and WAL effects, and the crossover between them.

Acknowledgments. This work was supported by the National Research Foundation of Korea (NRF) grant funded by the Korea government (MSIT) (No. 2018R1A2B6007837) and Creative-Pioneering Researchers Program through Seoul National University (SNU).
[1] P. W. Anderson, Absence of diffusion in certain random lattices, Phys. Rev. 109, 1492 (1958).

[2] P. A. Lee and T. V. Ramakrishnan, Disordered electronic systems, Rev. Mod. Phys. 57, 287 (1985).

[3] S. Datta, Electronic Transport in Mesoscopic Systems (Cambridge University Press, Cambridge, UK, 1995).

[4] E. Akkermans and G. Montambaux, Mesoscopic Physics of Electrons and Photons (Cambridge University Press, Cambridge, UK, 2007).

[5] S. Hikami, A. I. Larkin, and Y. Nagaoka, Spin-orbit interaction and magnetoresistance in the two dimensional random system, Prog. Theor. Phys. 63, 707 (1980).

[6] B. L. Altshuler, D. Khmel'nitzkii, A. I. Larkin, and P. A. Lee, Magnetoresistance and Hall effect in a disordered two-dimensional electron gas, Phys. Rev. B 22, 5142 (1984).

[7] P. Wölfle and R. N. Bhatt, Electron localization in anisotropic systems, Phys. Rev. B 30, 3542 (1984).

[8] D. E. Beutler and N. Giordano, Localization and electronelectron interaction effects in thin Bi wires and films, Phys. Rev. B 38, 8 (1988).

[9] G. Bergmann, Influence of Spin-Orbit Coupling on Weak Localization, Phys. Rev. Lett. 48, 1046 (1982).

[10] G. Bergmann, Weak localization in thin films: A time-offlight experiment with conduction electrons, Phys. Rep. 107, 1 (1984).

[11] H. Suzuura and T. Ando, Crossover from Symplectic to Orthogonal Class in a Two-Dimensional Honeycomb Lattice, Phys. Rev. Lett. 89, 266603 (2002).

[12] E. McCann, K. Kechedzhi, V. I. Fal'ko, H. Suzuura, T. Ando, and B. L. Altshuler, Weak-Localization Magnetoresistance and Valley Symmetry in Graphene, Phys. Rev. Lett. 97, 146805 (2006).

[13] R. V. Gorbachev, F. V. Tikhonenko, A. S. Mayorov, D. W. Horsell, and A. K. Savchenko, Weak Localization in Bilayer Graphene, Phys. Rev. Lett. 98, 176805 (2007)

[14] K. Kechedzhi, V. I. Fal'ko, E. McCann, and B. L. Altshuler, Influence of Trigonal Warping on Interference Effects in Bilayer Graphene, Phys. Rev. Lett. 98, 176806 (2007).

[15] K. Nomura, M. Koshino, and S. Ryu, Topological Delocalization of Two-Dimensional Massless Dirac Fermions, Phys. Rev. Lett. 99, 146806 (2007).

[16] K.-I. Imura, Y. Kuramoto, and K. Nomura, Weak localization properties of the doped $Z_{2}$ topological insulator, Phys. Rev. B 80, 085119 (2009).
[17] H.-Z. Lu and S.-Q. Shen, Weak antilocalization and localization in disordered and interacting Weyl semimetals, Phys. Rev. B 92, 035203 (2015).

[18] X. Dai, H.-Z. Lu, S.-Q. Shen, and H. Yao, Detecting monopole charge in Weyl semimetals via quantum interference transport, Phys. Rev. B 93, 161110(R) (2016).

[19] H.-Z. Lu and S.-Q. Shen, Quantum transport in topological semimetals under magnetic fields, Front. Phys. 12, 127201 (2017).

[20] W. Chen, H.-Z. Lu, and O. Zilberberg, Weak Localization and Antilocalization in Nodal-Line Semimetals: Dimensionality and Topological Effects, Phys. Rev. Lett. 122, 196603 (2019).

[21] B. Fu, H.-W. Wang, and S.-Q. Shen, Quantum Interference Theory of Magnetoresistance in Dirac Materials, Phys. Rev. Lett. 122, 246601 (2019).

[22] H.-T. He, G. Wang, T. Zhang, I.-K. Sou, G. K. L. Wong, and J.-N. Wang, Impurity Effect on Weak Antilocalization in the Topological Insulator $\mathrm{Bi}_{2} \mathrm{Te}_{3}$, Phys. Rev. Lett. 106, 166805 (2011).

[23] H.-Z. Lu, J. Shi, and S.-Q. Shen, Competition between Weak Localization and Antilocalization in Topological Surface States, Phys. Rev. Lett. 107, 076801 (2011).

[24] H. B. Zhang, H. L. Yu, D. H. Bao, S. W. Li, C. X. Wang, and G. W. Yang, Weak localization bulk state in a topological insulator $\mathrm{Bi}_{2} \mathrm{Te}_{3}$ film, Phys. Rev. B 86, 075102 (2012).

[25] A. S. Rodin, A. Carvalho, and A. H. Castro Neto, StrainInduced Gap Modification in Black Phosphorus, Phys. Rev. Lett. 112, 176801 (2014).

[26] A. N. Rudenko and M. I. Katsnelson, Quasiparticle band structure and tight-binding model for single- and bilayer black phosphorus, Phys. Rev. B 89, 201408 (2014).

[27] Q. Liu, X. Zhang, L. B. Abdalla, A. Fazzio, and A. Zunger, Switching a normal insulator into a topological insulator via electric field with application to phosphorene, Nano Lett. 15, 1222 (2015).

[28] S. Yuan, A. N. Rudenko, and M. I. Katsnelson, Transport and optical properties of single- and bilayer black phosphorus with defects, Phys. Rev. B 91, 115436 (2015).

[29] J. Kim, S. S. Baik, S. H. Ryu, Y. Sohn, S. Park, B.-G. Park, J. Denlinger, Y. Yi, H. J. Choi, and K. S. Kim, Observation of tunable band gap and anisotropic Dirac semimetal state in black phosphorus, Science 349, 723 (2015).

[30] J. M. Pereira, Jr. and M. I. Katsnelson, Landau levels of singlelayer and bilayer phosphorene, Phys. Rev. B 92, 075437 (2015). 
[31] S. S. Baik, K. S. Kim, Y. Yi, and H. J. Choi, Emergence of twodimensional massless Dirac fermions, chiral pseudospins, and Berry's phase in potassium doped few-layer black phosphorus, Nano Lett. 15, 7788 (2015).

[32] P. Adroguer, D. Carpentier, G. Montambaux, and E. Orignac, Diffusion of Dirac fermions across a topological merging transition in two dimensions, Phys. Rev. B 93, 125113 (2016).

[33] S. Yuan, E. van Veen, M. I. Katsnelson, and R. Roldán, Quantum Hall effect and semiconductor-to-semimetal transition in biased black phosphorus, Phys. Rev. B 93, 245433 (2016).

[34] H. Doh and H. J. Choi, Dirac-semimetal phase diagram of twodimensional black phosphorus, 2D Mater. 4, 025071 (2017).

[35] J. Kim, S. S. Baik, S. W. Jung, Y. Sohn, S. H. Ryu, H. J. Choi, B.-J. Yang, and K. S. Kim, Two-Dimensional Dirac Fermions Protected by Space-Time Inversion Symmetry in Black Phosphorus, Phys. Rev. Lett. 119, 226801 (2017).

[36] S. Park, S. Woo, and H. Min, Semiclassical Boltzmann transport theory of few-layer black phosphorous in various phases, 2D Mater. 6, 025016 (2019).

[37] J. Jang, S. Ahn, and H. Min, Optical conductivity of black phosphorus with a tunable electronic structure, 2D Mater. 6, 025029 (2019).

[38] L. Li, Y. Yu, G. J. Ye, Q. Ge, X. Ou, H. Wu, D. Feng, X. H. Chen, and Y. Zhang, Black phosphorus field-effect transistors, Nat. Nanotechnol. 9, 372 (2014).

[39] F. Xia, H. Wang, and Y. Jia, Rediscovering black phosphorus as an anisotropic layered material for optoelectronics and electronics, Nat. Commun. 5, 4458 (2014).

[40] V. Tran, R. Soklaski, Y. Liang, and L. Yang, Layer-controlled band gap and anisotropic excitons in few-layer black phosphorus, Phys. Rev. B 89, 235319 (2014).

[41] J. Qiao, X. Kong, Z.-X. Hu, F. Yang, and W. Ji, High-mobility transport anisotropy and linear dichroism in few-layer black phosphorus, Nat. Commun. 5, 4475 (2014).

[42] Z. J. Xiang, G. J. Ye, C. Shang, B. Lei, N. Z. Wang, K. S. Yang, D. Y. Liu, F. B. Meng, X. G. Luo, L. J. Zou, Z. Sun, Y. Zhang, and X. H. Chen, Pressure-Induced Electronic Transition in Black Phosphorus, Phys. Rev. Lett. 115, 186403 (2015).
[43] Y. Du, A. T. Neal, H. Zhou, and P. D. Ye, Weak localization in few-layer black phosphorus, 2D Mater. 3, 024003 (2016).

[44] Y. Shi, N. Gillgren, T. Espiritu, S. Tran, J. Yang, K. Watanabe, T. Taniguchi, and C. N. Lau, Weak localization and electronelectron interactions in few layer black phosphorus devices, $2 \mathrm{D}$ Mater. 3, 034003 (2016).

[45] N. Hemsworth, V. Tayari, F. Telesio, S. Xiang, S. Roddaro, M. Caporali, A. Ienco, M. Serrano-Ruiz, M. Peruzzini, G. Gervais, T. Szkopek, and S. Heun, Dephasing in strongly anisotropic black phosphorus, Phys. Rev. B 94, 245404 (2016).

[46] C.-H. Li, Y.-J. Long, L.-X. Zhao, L. Shan, Z.-A. Ren, J.-Z. Zhao, H.-M. Weng, X. Dai, Z. Fang, C. Ren, and G.-F. Chen, Pressure-induced topological phase transitions and strongly anisotropic magnetoresistance in bulk black phosphorus, Phys. Rev. B 95, 125417 (2017).

[47] G. D. Mahan, Many-Particle Physics, 3rd ed. (Springer, Berlin, 2000).

[48] P. Coleman, Introduction to Many-Body Physics (Cambridge University Press, Cambridge, UK, 2016).

[49] N. W. Ashcroft and N. D. Mermin, Solid State Physics (Brooks Cole, Pacific Grove, CA, 1976).

[50] S. Park, S. Woo, E. J. Mele, and H. Min, Semiclassical Boltzmann transport theory for multi-Weyl semimetals, Phys. Rev. B 95, 161113(R) (2017).

[51] S. Kim, S. Woo, and H. Min, Vertex corrections to the dc conductivity in anisotropic multiband systems, Phys. Rev. B 99, 165107 (2019).

[52] See Supplemental Material at http://link.aps.org/supplemental/ 10.1103/PhysRevResearch.2.022045 for the detailed derivations of the Cooperon ansatz, WL and WAL corrections, magnetoconductivity, and the discussion on the applicability of the generalized WL theory to other anisotropic multiband and multivalley systems.

[53] P. Dietl, F. Piéchon, and G. Montambaux, New Magnetic Field Dependence of Landau Levels in a Graphenelike Structure, Phys. Rev. Lett. 100, 236405 (2008).

[54] F. J. Dyson, Statistical theory of the energy levels of complex systems. I, J. Math. Phys. (NY) 3, 140 (1962). 